\title{
Iteration of certain meromorphic functions with unbounded singular values
}

\author{
TARAKANTA NAYAK $\dagger$ and M. GURU PREM PRASAD $\ddagger$ \\ $\dagger$ Department of Mathematics, National Institute of Technology Rourkela, \\ Rourkela 769008, India \\ (e-mail: tarakanta.nayak@nitrkl.ac.in) \\ \$ Department of Mathematics, Indian Institute of Technology Guwahati, \\ Guwahati 781039, India \\ (e-mail: mgpp@iitg.ac.in)
}

(Received 8 October 2008 and accepted in revised form 20 March 2009)

\begin{abstract}
Let $\mathcal{M}=\left\{f(z)=\left(z^{m} / \sinh ^{m} z\right)\right.$ for $z \in \mathbb{C} \mid$ either $m$ or $m / 2$ is an odd natural number\}. For each $f \in \mathcal{M}$, the set of singularities of the inverse function of $f$ is an unbounded subset of the real line $\mathbb{R}$. In this paper, the iteration of functions in oneparameter family $\mathcal{S}=\left\{f_{\lambda}(z)=\lambda f(z) \mid \lambda \in \mathbb{R} \backslash\{0\}\right\}$ is investigated for each $f \in \mathcal{M}$. It is shown that, for each $f \in \mathcal{M}$, there is a critical parameter $\lambda^{*}>0$ depending on $f$ such that a period-doubling bifurcation occurs in the dynamics of functions $f_{\lambda}$ in $\mathcal{S}$ when the parameter $|\lambda|$ passes through $\lambda^{*}$. The non-existence of Baker domains and wandering domains in the Fatou set of $f_{\lambda}$ is proved. Further, it is shown that the Fatou set of $f_{\lambda}$ is infinitely connected for $0<|\lambda| \leq \lambda^{*}$ whereas for $|\lambda| \geq \lambda^{*}$, the Fatou set of $f_{\lambda}$ consists of infinitely many components and each component is simply connected.
\end{abstract}

\section{Introduction}

Let $f: \mathbb{C} \rightarrow \widehat{\mathbb{C}}=\mathbb{C} \cup\{\infty\}$ be a non-constant transcendental meromorphic function. The set of points $z \in \widehat{\mathbb{C}}$ for which the sequence of iterates $\left\{f^{n}(z)\right\}_{n=0}^{\infty}$ is defined and forms a normal family is called the Fatou set of $f$ and is denoted by $\mathcal{F}(f)$. The Julia set, denoted by $\mathcal{J}(f)$, is the complement of the Fatou set of $f$ in $\widehat{\mathbb{C}}$. It is well known that the Fatou set is open and the Julia set is a perfect set. Let $\operatorname{sing}\left(f^{-1}\right)$ denote the set of finite singularities of the inverse function $f^{-1}$ of the function $f$ (also called singular values of $f$ ). Then, $\operatorname{sing}\left(f^{-1}\right)$ is the set of critical and finite asymptotic values of $f$ and finite limit points of these values. Denote by $\operatorname{sing}\left(f^{-p}\right)$ the set of finite singularities of the inverse function of $f^{p}$. Let $A_{k}(f)=\left\{z \in \mathbb{C} \mid f^{k}\right.$ is not analytic at $\left.z\right\}$ and define

$$
S_{p}(f)=\bigcup_{k=0}^{p-1} f^{k}\left(\operatorname{sing}\left(f^{-1}\right) \backslash A_{k}(f)\right) \quad \text { and } \quad P(f)=\bigcup_{p=1}^{\infty} S_{p}(f) .
$$


It is easy to see that $\operatorname{sing}\left(f^{-p}\right) \subseteq S_{p}(f) \subseteq S_{p+1}(f)$ and the set $P(f)$ consists of the forward orbits of all points in $\operatorname{sing}\left(f^{-1}\right)$ as long as they are defined and finite. Let $B$ denote the class of all meromorphic functions $f$ for which $\operatorname{sing}\left(f^{-1}\right)$ is a bounded set.

The existence of Baker domains and wandering domains is one of the important dynamical aspects of transcendental meromorphic functions and has been investigated $[1,5,6,8,15,16,18,20,23]$. Rippon and Stallard proved the non-existence of Baker domains with period $p$ in the Fatou set of transcendental meromorphic functions $f$ for which the set $S_{p}(f)$ is bounded [19]. Non-existence of wandering domains for meromorphic functions $f$ of finite type (i.e., $f$ for which $\operatorname{sing}\left(f^{-1}\right)$ is a finite set) is established by Baker et al [3]. A number of one-parameter families of meromorphic functions of finite type are investigated by Keen and Kotus [9], Keen et al [14], Jiang [13] and Prasad et al [11]. Zheng [22, 23] investigated the relations between $P(f)$ and the limit functions of iterates $\left\{f^{n}\right\}_{n>0}$ in a Fatou component and proved the non-existence of Baker domains and wandering domains for certain meromorphic functions in the class $B$. However, the dynamics of meromorphic functions outside the class $B$ is largely unexplored.

Let

$$
\mathcal{M}=\left\{f(z)=\frac{z^{m}}{\sinh ^{m} z} \text { for } z \in \mathbb{C} \mid m \text { or } m / 2 \text { is an odd natural number }\right\} .
$$

For each $f \in \mathcal{M}$, consider the one-parameter family of functions

$$
\mathcal{S}=\left\{f_{\lambda}(z)=\lambda f(z) \mid \lambda \in \mathbb{R} \backslash\{0\}\right\} .
$$

In this paper, the iteration of functions $f_{\lambda}$ in the one-parameter family $\mathcal{S}$ is investigated.

Observe that $f_{\lambda}(z)$ is an even function. If $\lambda \in \mathbb{R} \backslash\{0\}$ then $f_{\lambda}(z)=-f_{-\lambda}(-z)$ and $f_{\lambda}^{n}(z)=-f_{-\lambda}^{n}(-z)$ for $z \in \mathbb{C}$ and $n \in \mathbb{N}$. It shows that the functions $f_{\lambda}$ and $f_{-\lambda}$ are conformally conjugate and the dynamics of $f_{\lambda}$ and $f_{-\lambda}$ are essentially same. Therefore, we prove the results on the dynamics of the functions $f_{\lambda} \in \mathcal{S}$ for $\lambda>0$.

In $\S 2$, it is mainly shown that $\operatorname{sing}\left(f_{\lambda}^{-1}\right)$ is an unbounded subset of the real line. The dynamics of $f_{\lambda}(x)$ for $x \in \mathbb{R}$ is investigated in $\S 3$. We show that there is a critical parameter $\lambda^{*}>0$ (depending on $f$ ) such that a period-doubling bifurcation occurs in the dynamics of functions $f_{\lambda}$ in $\mathcal{S}$ when the parameter $|\lambda|$ passes through $\lambda^{*}$. In $\S 4$, the dynamics of $f_{\lambda}(z)$ for $z \in \mathbb{C}$ is studied. The non-existence of Baker domains and wandering domains in the Fatou set of $f_{\lambda}$ is also proved. There is a change in topology of the Fatou components effectuated by the above mentioned bifurcation which is described in $\S 5$.

\section{Properties of $f_{\lambda}$}

The function $f_{\lambda}(z)=\lambda\left(z^{m} / \sinh ^{m} z\right)$ is meromorphic with poles at $\{i \pi k \mid k \in \mathbb{Z} \backslash\{0\}\}$. All the poles are multiple if $m>1$ and simple if $m=1$. Further, the function $f_{\lambda}(z)$ is even and not periodic. In Proposition 2.1, we prove that the Julia set of $f_{\lambda}$ is symmetric with respect to both the real and imaginary axes. The point $z=0$ is an omitted value of $f_{\lambda}$ and hence an asymptotic value of $f_{\lambda}(z)$. More importantly, it is shown that $\operatorname{sing}\left(f_{\lambda}^{-1}\right)$ is an unbounded subset of the real line in Proposition 2.2.

Proposition 2.1. Let $f_{\lambda} \in \mathcal{S}$. If $z \in \mathcal{J}\left(f_{\lambda}\right)$ then $-z \in \mathcal{J}\left(f_{\lambda}\right)$ and $\bar{z} \in \mathcal{J}\left(f_{\lambda}\right)$. 
Proof. Let $z \in \mathcal{J}\left(f_{\lambda}\right)$. Since $f_{\lambda}(-z)=f_{\lambda}(z)$ for all $z \in \mathbb{C}$ and $\mathcal{J}\left(f_{\lambda}\right)$ is completely invariant, $-z \in \mathcal{J}\left(f_{\lambda}\right)$. Observe that $f_{\lambda}(\bar{z})=\overline{f_{\lambda}(z)}$ and consequently, $f_{\lambda}^{n}(\bar{z})=\overline{f_{\lambda}^{n}(z)}$ for all $z \in \mathbb{C}$ and $n \in \mathbb{N}$. For $z \in \mathcal{J}\left(f_{\lambda}\right)$, the sequence $\left\{f_{\lambda}^{n}\right\}_{n>0}$ is not normal at $z$. It follows that $\left\{\bar{f}_{\lambda}^{n}\right\}_{n>0}$ is also not normal at $z$. Therefore, $\left\{f_{\lambda}^{n}\right\}_{n>0}$ is not normal at $\bar{z}$ and $\bar{z} \in \mathcal{J}\left(f_{\lambda}\right)$.

Proposition 2.2. Let $f_{\lambda} \in \mathcal{S}$. Then, the set of all the critical values of $f_{\lambda}$ is an unbounded subset of $\mathbb{R} \backslash(-|\lambda|,|\lambda|)$ and 0 is the only finite asymptotic value of $f_{\lambda}$.

Proof. Observe that

$$
f_{\lambda}^{\prime}(z)=\lambda \frac{m z^{m-1}}{\sinh ^{m-1} z}\left\{\frac{\sinh z-z \cosh z}{\sinh ^{2} z}\right\} \text { and } \frac{m z^{m-1}}{\sinh ^{m-1} z} \neq 0 \quad \text { for } z \in \mathbb{C} .
$$

Further, the point $z=0$ is the only common zero of $\sinh z-z \cosh z$ and $\sinh ^{2} z$ and is a zero of $(\sinh z-z \cosh z) / \sinh ^{2} z$. Therefore, the solutions of $f_{\lambda}^{\prime}(z)=0$ are precisely the solutions of $\sinh z-z \cosh z=0$ i.e., the solutions of $\tanh z=z$. It is easy to see that the set of all the solutions of $\tanh z=z$ is an unbounded subset of the imaginary axis. If $\tanh (i y)=i y$ for some $y \in \mathbb{R}$ then $\tanh (-i y)=-\tanh (i y)=-i y$. Therefore, the set of all the critical points of $f_{\lambda}(z)$ is symmetric with respect to the origin and is an unbounded subset of the imaginary axis. Let $\left\{i y_{k}\right\}_{k>0}$ be the sequence of critical points in the positive imaginary axis arranged in the increasing order of their moduli. Then $-i y_{k}$ is also a critical point of $f_{\lambda}(z)$ for each $k$. Since $f_{\lambda}(z)$ is an even function,

$$
\lim _{k \rightarrow \infty}\left|f_{\lambda}\left(i y_{k}\right)\right|=\lim _{k \rightarrow \infty}\left|f_{\lambda}\left(-i y_{k}\right)\right|=\lim _{k \rightarrow \infty}\left|\lambda \frac{i^{m} y_{k}^{m}}{i^{m} \sin ^{m} y_{k}}\right|=\infty \text {. }
$$

Therefore, the set of all the critical values of $f_{\lambda}$ is unbounded. Every critical point $i y_{k}$ of $f_{\lambda}(z)$ satisfies $\tanh \left(i y_{k}\right)=i y_{k}$ and consequently,

$$
\frac{i y_{k}}{\sinh \left(i y_{k}\right)}=\frac{1}{\cosh \left(i y_{k}\right)} \text {. }
$$

The critical value

$$
f_{\lambda}\left(i y_{k}\right)=\lambda\left(\frac{i y_{k}}{\sinh \left(i y_{k}\right)}\right)^{m}=\lambda\left(\frac{1}{\cosh \left(i y_{k}\right)}\right)^{m}=\lambda\left(\frac{1}{\cos y_{k}}\right)^{m}
$$

is real. Since $|\cos y| \leq 1$ for all $y \in \mathbb{R}$, it follows that $\left|f_{\lambda}\left(i y_{k}\right)\right| \geq|\lambda|$. Therefore, the set of all the critical values of $f_{\lambda}(z)$ is an unbounded subset of $\mathbb{R} \backslash(-|\lambda|,|\lambda|)$.

In order to determine the asymptotic values of $f_{\lambda}$, first we find all the asymptotic values of $(\sinh z / z)$. All the critical points of $(\sinh z / z)$, i.e., the roots of $(z \cosh z-\sinh z) / z^{2}$ are purely imaginary and form an unbounded set. Since

$$
\lim _{|y| \rightarrow \infty} \frac{\sinh i y}{i y}=\lim _{|y| \rightarrow \infty} \frac{\sin y}{y}=0,
$$

0 is an asymptotic value of $(\sinh z / z)$ and is the only limit point of all the critical values of $(\sinh z / z)$. Since the order of $(\sinh z / z)$ is one, it can have at most two finite asymptotic values. Further, if there are exactly two finite asymptotic values of $(\sinh z / z)$ then both the asymptotic values are indirect singularities of the inverse function of $(\sinh z / z)$ [17]. If $f$ is 
a meromorphic function of finite order and $a$ is an asymptotic value of $f$ then, $a$ is a limit point of critical values $a_{k} \neq a$ or all singularities of $f^{-1}$ are logarithmic (a special case of direct singularity) [7]. Therefore, if there is a finite asymptotic value $\hat{w}$ of $(\sinh z / z)$ other than 0 then both 0 and $\hat{w}$ are indirect singularities of inverse function of $(\sinh z / z)$ and the limit points of critical values of $(\sinh z / z)$. Since the critical values of $(\sinh z / z)$ accumulate only at $0, \hat{w}$ can not be an asymptotic value of $(\sinh z / z)$. Thus, 0 is the only finite asymptotic value of $(\sinh z / z)$. Since $(\sinh z / z)$ is an entire function, $\infty$ is also an asymptotic value. It implies that the function $(z / \sinh z)$ has only one finite asymptotic value, namely 0 . Hence, 0 is the only finite asymptotic value of $f_{\lambda}(z)=\lambda\left(z^{m} / \sinh ^{m} z\right)$ for $m \in \mathbb{N}$.

Remark 2.1. For $z=x+i y \neq 0$,

$$
\left|\frac{z^{m}}{\sinh ^{m} z}\right|=\frac{|z|^{m}}{|\sinh z|^{m}}=\left\{\left(\frac{x^{2}+y^{2}}{\sinh ^{2} x+\sin ^{2} y}\right)^{1 / 2}\right\}^{m} .
$$

If $\gamma:[0, \infty) \rightarrow \mathbb{C}$ is a path for which $\{\Im(z) \mid z \in \gamma\}$ is bounded and $\lim _{t \rightarrow \infty}|\Re(\gamma(t))|=$ $\infty$ then $\lim _{t \rightarrow \infty} f_{\lambda}(\gamma(t))=0$. Further, if $\gamma$ is a path for which $\{\Re(z) \mid z \in \gamma\}$ is bounded and $\lim _{t \rightarrow \infty}|\mathfrak{\Im}(\gamma(t))|=\infty$ then $\lim _{t \rightarrow \infty} f_{\lambda}(\gamma(t))=\infty$.

\section{Dynamics of $f_{\lambda}(x)$ for $x \in \mathbb{R}$}

In this section, the dynamics of $f_{\lambda}(x)$ for $x \in \mathbb{R}$ is studied. In Theorem 3.1, the existence and nature of real fixed points of $f_{\lambda}$ are explored. The change in the nature and existence of real periodic points leads to a bifurcation in the dynamics of $f_{\lambda}(x)$ for $x \in \mathbb{R}$ at a critical parameter value and is proved in Theorem 3.2.

Consider the function

$$
\begin{aligned}
\phi(x) & =x f^{\prime}(x)+f(x)=x \frac{m x^{m-1}}{\sinh ^{m+1} x}(\sinh x-x \cosh x)+\frac{x^{m}}{\sinh ^{m}(x)} \\
& =\frac{x^{m}}{\sinh ^{m+1}(x)}((m+1) \sinh x-m x \cosh x) \quad \text { for } x \geq 0 .
\end{aligned}
$$

Let $p(x)=(m+1) \sinh x-m x \cosh x$. Then $p^{\prime}(x)=\cosh x-m x \sinh x$ and

$$
p^{\prime \prime}(x)=(1-m) \sinh x-m x \cosh x .
$$

Observe that $p^{\prime \prime}(x)<0$ for $x \in \mathbb{R}^{+}=\{x \in \mathbb{R} \mid x>0\}$, since $m \geq 1$. Therefore, the function $p^{\prime}(x)$ is decreasing on $\mathbb{R}^{+}$. Since $p^{\prime}(0)=1$ and $\lim _{x \rightarrow+\infty} p^{\prime}(x)=-\infty$, by continuity of $p^{\prime}(x)$, it follows that there is a unique $\hat{x}>0$ such that $p^{\prime}(x)>0$ for $0 \leq x<\hat{x}, p^{\prime}(\hat{x})=0$ and $p^{\prime}(x)<0$ for $x>\hat{x}$. Therefore, $p(x)$ increases in $[0, \hat{x})$, attains its maximum at $\hat{x}$ and decreases thereafter. It follows from the facts $p(0)=0$ and $\lim _{x \rightarrow+\infty} p(x)=-\infty$ that, there is a unique positive $x^{*}>\hat{x}$ such that $p(x)>0$ for $0<x<x^{*}, p\left(x^{*}\right)=0$ and $p(x)<0$ for $x>x^{*}$. Since $\left(x^{m} / \sinh ^{m+1} x\right)>0$ for all $x>0$, it follows that

$$
\phi(x)=\frac{x^{m}}{\sinh ^{m+1} x} p(x) \begin{cases}>0 & \text { for } 0<x<x^{*}, \\ =0 & \text { for } x=x^{*} \\ <0 & \text { for } x>x^{*}\end{cases}
$$


Define

$$
\lambda^{*}(m)=\lambda^{*}=\frac{x^{*}}{f\left(x^{*}\right)}
$$

where $x^{*}$ is the unique positive real root of the equation $\phi(x)=x f^{\prime}(x)+f(x)=0$.

Remark 3.1. For the function $f(x)=\left(x^{m} / \sinh ^{m} x\right)$, let $x^{*}(m)$ denote the positive real root of the equation $\phi(x)=x f^{\prime}(x)+f(x)=0$ and let

$$
\lambda^{*}(m)=\frac{x^{*}(m)}{f\left(x^{*}(m)\right)}
$$

denote the corresponding critical parameter. For $m=1,2$ and 3 , it is numerically computed that $x^{*}(1) \approx 1.915, x^{*}(2) \approx 1.2878, x^{*}(3) \approx 1.03402$ and $\lambda^{*}(1) \approx 3.3198$, $\lambda^{*}(2) \approx 2.1772, \lambda^{*}(3) \approx 1.7926$.

The following theorem shows that $f_{\lambda}$ has a unique real fixed point for each $\lambda>0$. However, the nature of the fixed point changes when the parameter $\lambda$ passes through the critical parameter $\lambda^{*}$.

THEOREM 3.1. Let $f_{\lambda} \in \mathcal{S}$ and $\lambda>0$. Then, the function $f_{\lambda}$ has a unique real fixed point $x_{\lambda}$. Furthermore, the following cases hold.

(1) The fixed point $x_{\lambda}$ is attracting for $0<\lambda<\lambda^{*}$.

(2) The fixed point $x_{\lambda}$ is rationally indifferent for $\lambda=\lambda^{*}$.

(3) The fixed point $x_{\lambda}$ is repelling for $\lambda>\lambda^{*}$.

Proof. Since $f_{\lambda}(x)>0$ for all $x \in \mathbb{R}$, each real periodic point of $f_{\lambda}$ is positive. The function

$$
f_{\lambda}^{\prime}(x)=\lambda \frac{m x^{m-1}}{\sinh ^{m+1} x}(\sinh x-x \cosh x)<0 \quad \text { for } x>0
$$

and hence $f_{\lambda}(x)$ is decreasing on $\mathbb{R}^{+}$. Let $g_{\lambda}(x)=f_{\lambda}(x)-x$ for $x \in \mathbb{R}$. Since $f_{\lambda}^{\prime}(x)<0$ for $x>0, g_{\lambda}^{\prime}(x)=f_{\lambda}^{\prime}(x)-1<0$ and consequently, $g_{\lambda}(x)$ is decreasing on $\mathbb{R}^{+}$. Now, $g_{\lambda}(0)=\lambda>0, \lim _{x \rightarrow+\infty} g_{\lambda}(x)=-\infty$ and $g_{\lambda}(x)$ is continuous on $\mathbb{R}^{+}$. By the intermediate-value theorem, there exists a unique positive $x_{\lambda}$ such that $g_{\lambda}\left(x_{\lambda}\right)=0$. In other words, $f_{\lambda}(x)$ has a unique positive fixed point $x_{\lambda}$ and $\lambda=\left(x_{\lambda} / f\left(x_{\lambda}\right)\right)$. Note that the function $(x / f(x))$ is increasing on $\mathbb{R}^{+}$, since

$$
\frac{d}{d x}\left(\frac{x}{f(x)}\right)=\frac{f(x)-x f^{\prime}(x)}{(f(x))^{2}}>0 \text { for } x>0 .
$$

(1) For $0<\lambda<\lambda^{*},\left(x_{\lambda} / f\left(x_{\lambda}\right)\right)<\left(x^{*} / f\left(x^{*}\right)\right)$ which gives $x_{\lambda}<x^{*}$. By equation (2), $\phi\left(x_{\lambda}\right)>0$. This implies that

$$
\frac{\phi\left(x_{\lambda}\right)}{f\left(x_{\lambda}\right)}=\frac{x f^{\prime}\left(x_{\lambda}\right)+f\left(x_{\lambda}\right)}{f\left(x_{\lambda}\right)}=f_{\lambda}^{\prime}\left(x_{\lambda}\right)+1>0 .
$$

Since $f_{\lambda}^{\prime}(x)$ is negative on $\mathbb{R}^{+}$, it follows that $-1<f_{\lambda}^{\prime}\left(x_{\lambda}\right)<0$ and the fixed point $x_{\lambda}$ is attracting for $0<\lambda<\lambda^{*}$.

(2) For $\lambda=\lambda^{*}$, it follows that $x_{\lambda}=x^{*}$ and $\phi\left(x_{\lambda}\right)=0$ by arguments similar to those used in case (1). Now, by equation (2), it follows that $\left(\phi\left(x_{\lambda}\right) / f\left(x_{\lambda}\right)\right)=0$ implying $f_{\lambda^{*}}^{\prime}\left(x_{\lambda}\right)=-1$. Therefore, the fixed point $x_{\lambda}=x^{*}$ is rationally indifferent if $\lambda=\lambda^{*}$. 
(3) For $\lambda>\lambda^{*}$, it follows that $x_{\lambda}>x^{*}$ by arguments similar to those used in case (1). Again by equation (2) and by the fact $x_{\lambda}>x^{*}$, we have $\phi\left(x_{\lambda}\right)<0$. It shows that $\left(\phi\left(x_{\lambda}\right) / f\left(x_{\lambda}\right)\right)=f_{\lambda}^{\prime}\left(x_{\lambda}\right)+1<0$ and hence $f_{\lambda}^{\prime}\left(x_{\lambda}\right)<-1$. Therefore, $x_{\lambda}$ is a repelling fixed point of $f_{\lambda}$ for $\lambda>\lambda^{*}$.

Now, we investigate the possibility of the real periodic points of $f_{\lambda}$ with minimal period greater than one. The function $f_{\lambda}(x)$ is decreasing on $\mathbb{R}^{+}, f_{\lambda}(\mathbb{R})=(0, \lambda]$ and $f_{\lambda}$ has a unique real fixed point $x_{\lambda}$ by Theorem 3.1. It is easy to see that $f_{\lambda}(0)=$ $\lambda>f_{\lambda}(x)>x_{\lambda}$ for $0<x<x_{\lambda}$ and $f_{\lambda}(x)<x_{\lambda}<f_{\lambda}(0)=\lambda$ for $x>x_{\lambda}>0$. In other words, $f_{\lambda}\left(\left(0, x_{\lambda}\right)\right)=\left(x_{\lambda}, \lambda\right)$ and $f_{\lambda}\left(x_{\lambda}, \infty\right)=\left(0, x_{\lambda}\right)$. It follows that $f_{\lambda}^{n}(x) \neq x$ for any $x \in \mathbb{R}^{+} \backslash\left\{x_{\lambda}\right\}$ and odd $n$. Therefore, $f_{\lambda}(x)$ does not have any real periodic point of odd period other than $x_{\lambda}$. Observe that $f_{\lambda}(x)>0$ and $f_{\lambda}^{\prime}(x)<0$ for $x>0$ and $\lambda>0$. So $\left(f_{\lambda}^{2}\right)^{\prime}(x)=f_{\lambda}^{\prime}\left(f_{\lambda}(x)\right) f_{\lambda}^{\prime}(x)>0$ and $f_{\lambda}^{2}(x)$ is increasing on $\mathbb{R}^{+}$. Consequently, if $f_{\lambda}^{2}(x)>$ $x$ (or $f_{\lambda}^{2}(x)<x$ ) for some $x \in \mathbb{R}^{+}$then $f_{\lambda}^{2 n}(x)>f_{\lambda}^{2(n-1)}(x)\left(\right.$ or $f_{\lambda}^{2 n}(x)<f_{\lambda}^{2(n-1)}(x)$ ) for all $n$. It shows that the function $f_{\lambda}^{2}(x)$ does not have any real periodic points of period greater than one, and hence $f_{\lambda}(x)$ has no real periodic point of even period greater than two. Therefore, a real periodic point of $f_{\lambda}$ other than $x_{\lambda}$ is of minimal period exactly equal to two, if it exists. Also, each cycle $\left\{x_{1 \lambda}, x_{2 \lambda}\right\}$ of real 2-periodic points satisfies $x_{1 \lambda}<x_{\lambda}<x_{2 \lambda}$. Let us assume that $f_{\lambda}$ has two different 2-periodic real cycles $\{a, b\}$ with $0<a<b$ and $\{c, d\}$ with $0<c<d$. Since $f_{\lambda}(x)$ is strictly decreasing on $\mathbb{R}^{+}$for $\lambda>0$, it follows that $c<a<x_{\lambda}<b<d$ or $a<c<x_{\lambda}<d<b$. In the first case $\{c, d\}$ and in the second case $\{a, b\}$ is called the outer cycle. In the first case $\{a, b\}$ and in the second case $\{c, d\}$ is called the inner cycle. The following proposition shows that whenever such a 2-periodic cycle exists, it is attracting or rationally indifferent and all the singular values of $f_{\lambda}(z)$ tend to this cycle under iteration of $f_{\lambda}^{2}$.

Proposition 3.1. Let $f_{\lambda} \in \mathcal{S}$ and $\lambda>0$. If $f_{\lambda}$ has a real 2-periodic cycle, then $\lim _{n \rightarrow \infty} f_{\lambda}^{2 n}(x)=y_{1 \lambda}$ or $y_{2 \lambda}$ for all $x \in\left[0, y_{1 \lambda}\right] \cup\left[y_{2 \lambda},+\infty\right)$ where $\left\{y_{1 \lambda}, y_{2 \lambda}\right\}$ is the outermost 2-periodic cycle. In particular, the cycle $\left\{y_{1 \lambda}, y_{2 \lambda}\right\}$ is either attracting or rationally indifferent and all the singular values of $f_{\lambda}$ tend to $\left\{y_{1 \lambda}, y_{2 \lambda}\right\}$ under iteration of $f_{\lambda}^{2}$.

Proof. It is observed earlier that any periodic point of the function $f_{\lambda}$ is of minimal period one or two and each 2-periodic cycle $\{a, b\}$ satisfies $a<x_{\lambda}<b$ where $x_{\lambda}$ is the fixed point of $f_{\lambda}$. Since $\left\{y_{1 \lambda}, y_{2 \lambda}\right\}$ is the outermost 2-periodic cycle, $f_{\lambda}^{2}(x) \neq x$ for all $x>y_{2 \lambda}$. If possible, let $f_{\lambda}^{2}(x)>x$ for some $x>y_{2 \lambda}$. Then, the sequence $\left\{f_{\lambda}^{2 n}(x)\right\}_{n>0}$ is increasing and bounded above by $\lambda$, and hence $f_{\lambda}^{2 n}(x)$ converges to $l$, say. Obviously, $l>y_{2 \lambda}$. By the continuity of $f_{\lambda}^{2}$ it follows that the point $l$ must be a periodic point of $f_{\lambda}$ of period at most two. This contradicts the fact that $\left\{y_{1 \lambda}, y_{2 \lambda}\right\}$ is the outermost 2-periodic cycle. Therefore, we conclude that $f_{\lambda}^{2}(x)<x$ for all $x>y_{2 \lambda}$. Since $f_{\lambda}^{2}(x)$ is increasing, the sequence $\left\{f_{\lambda}^{2 n}(x)\right\}_{n>0}$ is decreasing and bounded below by $y_{2 \lambda}$ and consequently, $\lim _{n \rightarrow \infty} f_{\lambda}^{2 n}(x)=$ $y_{2 \lambda}$ for $x>y_{2 \lambda}$. Similarly, it can be proved that $f_{\lambda}^{2}(x)>x$ and $\lim _{n \rightarrow \infty} f_{\lambda}^{2 n}(x)=y_{1 \lambda}$ for all $0 \leq x<y_{1 \lambda}$. Therefore, $\lim _{n \rightarrow \infty} f_{\lambda}^{2 n}(x)=y_{1 \lambda}$ or $y_{2 \lambda}$ for all $x \in\left[0, y_{1 \lambda}\right] \cup\left[y_{2 \lambda},+\infty\right)$.

Each interval containing $y_{1 \lambda}$ contains points tending to $y_{1 \lambda}$ under iteration of $f_{\lambda}^{2}$. Therefore, $y_{1 \lambda}$ cannot be a repelling periodic point of $f_{\lambda}^{2}$ and is either attracting or 
rationally indifferent. Thus, $\left\{y_{1 \lambda}, y_{2 \lambda}\right\}$ is either attracting or rationally indifferent. As $\left(-y_{2 \lambda}, y_{2 \lambda}\right) \subset(-\lambda, \lambda)$ and $f_{\lambda}$ is an even function, $\lim _{n \rightarrow \infty} f_{\lambda}^{2 n}(x)=y_{1 \lambda}$ or $y_{2 \lambda}$ for all $x \in \mathbb{R} \backslash(-\lambda, \lambda)$. Since all the critical values of $f_{\lambda}$ are in $\mathbb{R} \backslash(-\lambda, \lambda)$ and the finite asymptotic value 0 is mapped to $\lambda$ by $f_{\lambda}$, it is concluded that all the singular values of $f_{\lambda}$ tend to $\left\{y_{1 \lambda}, y_{2 \lambda}\right\}$ under iteration of $f_{\lambda}^{2}$.

The dynamics of $f_{\lambda}(x)$ for $x \in \mathbb{R}$ is determined in the following theorem.

THEOREM 3.2. Let $f_{\lambda} \in \mathcal{S}$ and $\lambda>0$.

(1) If $\lambda<\lambda^{*}$ then $\lim _{n \rightarrow \infty} f_{\lambda}^{n}(x)=a_{\lambda}$ for all $x \in \mathbb{R}$ where $a_{\lambda}$ is the unique real attracting fixed point of $f_{\lambda}$.

(2) If $\lambda=\lambda^{*}$ then $\lim _{n \rightarrow \infty} f_{\lambda}^{n}(x)=x^{*}$ for all $x \in \mathbb{R}$ where $x^{*}$ is the unique real rationally indifferent fixed point of $f_{\lambda}$.

(3) If $\lambda>\lambda^{*}$ then $\lim _{n \rightarrow \infty} f_{\lambda}^{2 n}(x)=a_{1 \lambda}$ or $a_{2 \lambda}$ for all $x \in \mathbb{R} \backslash\left\{r_{\lambda},-r_{\lambda}\right\}$ where $r_{\lambda}$ is the unique real repelling fixed point of $f_{\lambda}$ and $\left\{a_{1 \lambda}, a_{2 \lambda}\right\}$ is the real attracting or rationally indifferent 2-periodic cycle.

Proof. All the singular values of $f_{\lambda}(z)$ are in $(\mathbb{R} \backslash(-\lambda, \lambda)) \cup\{0\}$ by Proposition 2.2. If there is a 2-periodic cycle then the cycle is in $(0, \lambda)$ and by Proposition 3.1, all the singular values tend to the outermost 2-cycle under iteration of $f_{\lambda}^{2}$.

(1) Let $f_{\lambda}^{2}(x)>x$ (or $f_{\lambda}^{2}(x)<x$ ) for some $x>0$. Since $f_{\lambda}^{2}(x)$ is increasing on $\mathbb{R}^{+}$, the sequence $\left\{f_{\lambda}^{2 n}(x)\right\}_{n>0}$ is increasing and bounded above by $\lambda$ (or decreasing and bounded below by 0 ). Therefore, $f_{\lambda}^{2 n}(x)$ converges to $\hat{x}$, say. Now, by continuity of $f_{\lambda}$, the point $\hat{x}$ is a periodic point of $f_{\lambda}(x)$ of period one or two. If possible, let $\hat{x}$ be a periodic point of $f_{\lambda}$ with prime period two. Then, there is an outermost 2-periodic cycle of $f_{\lambda}$ and all the singular values of $f_{\lambda}$ tend to the outermost 2-periodic cycle under iteration of $f_{\lambda}^{2}$ which is a contradiction to the fact that the basin of attraction of $a_{\lambda}$ must contain at least one singular value of $f_{\lambda}$. Therefore, $\hat{x}$ is not a 2-periodic point and is a fixed point. Since $f_{\lambda}$ has only one real fixed point $a_{\lambda}$ for $0<\lambda<\lambda^{*}, \hat{x}=a_{\lambda}$ and $\lim _{n \rightarrow \infty} f_{\lambda}^{2 n}(x)=a_{\lambda}$ for all $x \in \mathbb{R}^{+}$. By continuity of $f_{\lambda}$, it follows that $\lim _{n \rightarrow \infty} f_{\lambda}^{n}(x)=a_{\lambda}$ for all $x \in \mathbb{R}^{+}$. Since

$$
f_{\lambda}\left(\mathbb{R}^{-} \cup\{0\}\right) \subset \mathbb{R}^{+}, \quad \lim _{n \rightarrow \infty} f_{\lambda}^{n}(x)=a_{\lambda} \quad \text { for all } x \in \mathbb{R} .
$$

(2) Let $f_{\lambda}^{2}(x)>x$ (or $f_{\lambda}^{2}(x)<x$ ). Since $f_{\lambda}^{2}(x)$ is increasing on $\mathbb{R}^{+}$, the sequence $\left\{f_{\lambda}^{2 n}(x)\right\}_{n>0}$ is increasing and bounded above by $\lambda$ (or decreasing and bounded below by 0 ). Proceeding as in case (1), it is easy to see that $\left\{f_{\lambda}^{2 n}(x)\right\}_{n>0}$ converges to $x^{*}$ for all $x \in \mathbb{R}^{+}$. By continuity of $f_{\lambda}$, it follows that $\lim _{n \rightarrow \infty} f_{\lambda}^{n}(x)=x^{*}$ for all $x \in \mathbb{R}^{+}$. Since

$$
f_{\lambda}\left(\mathbb{R}^{-} \cup\{0\}\right) \subset \mathbb{R}^{+}, \quad \lim _{n \rightarrow \infty} f_{\lambda}^{n}(x)=x^{*} \quad \text { for all } x \in \mathbb{R} .
$$

(3) If $\lambda>\lambda^{*}$, then the unique real fixed point of $f_{\lambda}$ is repelling. Therefore, we can find a real number $x$ sufficiently close to the fixed point $r_{\lambda}$ such that $f_{\lambda}^{2}(x)>x$. Since $f_{\lambda}^{2}(x)$ is increasing on $\mathbb{R}^{+}$, the sequence $\left\{f_{\lambda}^{2 n}(x)\right\}_{n>0}$ is increasing and bounded above by $\lambda$. Therefore, $\left\{f_{\lambda}^{2 n}(x)\right\}_{n>0}$ converges to $\hat{x}$, say. By continuity of $f_{\lambda}^{2}$, it follows that $\hat{x}$ is a 2-periodic point of $f_{\lambda}$. If possible, let there be more than one 2-periodic cycle of periodic points. If $\left\{i_{1 \lambda}, i_{2 \lambda}\right\}$ is the innermost real cycle of 2-periodic points of $f_{\lambda}$ then $i_{1 \lambda}<r_{\lambda}<i_{2 \lambda}$ and, $f_{\lambda}(x) \in\left(r_{\lambda}, i_{2 \lambda}\right)$ for all $x \in\left(i_{1 \lambda}, r_{\lambda}\right)$ and $f_{\lambda}(x) \in\left(i_{1 \lambda}, r_{\lambda}\right)$ 
for all $x \in\left(r_{\lambda}, i_{2 \lambda}\right)$. Furthermore, the sequence $\left\{f_{\lambda}^{2 n}(x)\right\}_{n>0}$ converges either to $i_{1 \lambda}$ or to $i_{2 \lambda}$ for $x \in\left(i_{1 \lambda}, i_{2 \lambda}\right) \backslash r_{\lambda}$ by the same arguments as used in the previous cases. Therefore, $\left\{i_{1 \lambda}, i_{2 \lambda}\right\}$ is either an attracting or a rationally indifferent cycle and at least one singular value of $f_{\lambda}$ tends to this cycle under iteration of $f_{\lambda}^{2}$. But all the singular values of $f_{\lambda}^{2}$ tend to the outermost 2-cycle under iteration of $f_{\lambda}$ by Proposition 3.1 leading to a contradiction. Hence, $f_{\lambda}$ has exactly one 2-periodic cycle. Let it be $\left\{a_{1 \lambda}, a_{2 \lambda}\right\}$. By Proposition 3.1, $\lim _{n \rightarrow \infty} f_{\lambda}^{2 n}(x)=a_{1 \lambda}$ or $a_{2 \lambda}$ for all $x \in\left[0, a_{1 \lambda}\right] \cup\left[a_{2 \lambda},+\infty\right)$. If $x \in\left(r_{\lambda}, a_{2 \lambda}\right]$, then $f_{\lambda}^{2}(x)>x$ and $\lim _{n \rightarrow \infty} f_{\lambda}^{2 n}(x)=a_{2 \lambda}$. Similarly, it is easily seen that $\lim _{n \rightarrow \infty} f_{\lambda}^{2 n}(x)=a_{1 \lambda}$ for all $x \in\left[a_{1 \lambda}, r_{\lambda}\right)$. Since $f_{\lambda}(z)$ is an even function, it follows that $\lim _{n \rightarrow \infty} f_{\lambda}^{2 n}(x)=a_{1 \lambda}$ or $a_{2 \lambda}$ for all $x \in \mathbb{R}^{-} \backslash\left\{-r_{\lambda}\right\}$. Therefore, if $\lambda>\lambda^{*}$ it is concluded that $\lim _{n \rightarrow \infty} f_{\lambda}^{2 n}(x)=a_{1 \lambda}$ or $a_{2 \lambda}$ for all $x \in \mathbb{R} \backslash\left\{r_{\lambda},-r_{\lambda}\right\}$ where $r_{\lambda}$ is the repelling fixed point of $f_{\lambda}$ and $\left\{a_{1 \lambda}, a_{2 \lambda}\right\}$ is the attracting or rationally indifferent 2-periodic cycle.

The above theorem exhibits the occurrence of a period-doubling bifurcation at $\lambda=\lambda^{*}$ in the dynamics of functions $f_{\lambda}$ in the one-parameter family $\mathcal{S}$.

Remark 3.2. All the singular values of $f_{\lambda}, \lambda>0$ are in $\mathbb{R}$ and tend to either an attracting or a rationally indifferent periodic point under iteration of $f_{\lambda}^{2}$. Therefore, the set $P\left(f_{\lambda}\right)$ is contained in the Fatou set of $f_{\lambda}$ for $\lambda>0$. In particular, the point 0 is in the Fatou set $\mathcal{F}\left(f_{\lambda}\right)$ for $\lambda>0$.

Remark 3.3. Note that $f_{\lambda}(i y)=\left(y^{m} / \sin ^{m} y\right)$ and the image of any point on the imaginary axis is either infinity or a real number. By Theorem 3.2, each of the real numbers except at most two are in an attracting or a parabolic domain of $f_{\lambda}$ corresponding to a real periodic point. Therefore, any Fatou component $U$ of $f_{\lambda}$ other than an attracting or parabolic domain (and their pre-images) intersects neither the real nor the imaginary axis. Thus, such a Fatou component $U$ is contained completely in one of the four quadrants of the complex plane.

\section{Dynamics of $f_{\lambda}(z)$ for $z \in \mathbb{C}$}

The dynamics of $f_{\lambda}(z)$ for $z \in \mathbb{C}$ is studied in this section. The non-existence of Baker domains and wandering domains in the Fatou set of $f_{\lambda} \in \mathcal{S}$ for $\lambda>0$ is proved in Theorem 4.1 and Theorem 4.2 respectively. The dynamics of $f_{\lambda}(z)$ for $z \in \mathbb{C}$ is described in Theorem 4.3.

THEOREM 4.1. Let $f_{\lambda} \in \mathcal{S}$ and $\lambda>0$. Then, the Fatou set of $f_{\lambda}$ has no Baker domain.

Proof. Suppose, on the contrary that the Fatou set of $f_{\lambda}$ has a Baker domain $B$ of minimal period $p$. All the singular values of $f_{\lambda}$ are real by Proposition 2.2 and $f_{\lambda}(\mathbb{R})=(0, \lambda]$. Therefore, $S_{p}\left(f_{\lambda}\right)$ is bounded for each $p>1$ and the Fatou set of $f_{\lambda}$ cannot have a Baker domain of minimal period greater than one [19]. Therefore, $p=1$. That is, $B$ is an invariant Baker domain. By the definition of an invariant Baker domain, there is a point $z^{*}$ in the boundary of $B$ such that $\lim _{n \rightarrow \infty} f_{\lambda}^{n}(z)=z^{*}$ for all $z \in B$ and $f_{\lambda}\left(z^{*}\right)$ is not defined. Since the point at infinity is the only point in $\widehat{\mathbb{C}}$ where the function $f_{\lambda}(z)$ is not defined, $z^{*}=\infty$. Now, $\lim _{n \rightarrow \infty} f_{\lambda}^{n}(z)=\infty$ and $f_{\lambda}^{n}(z) \in B$ for $z \in B$ and $n \in \mathbb{N}$ gives that the domain $B$ is unbounded. Since $f_{\lambda}(\bar{z})=\overline{f_{\lambda}(z)}$ for all $z \in \mathbb{C}$ and $B$ is contained in one of 
the four quadrants by Remark 3.3, $\bar{B}=\{\bar{z} \in \mathbb{C} \mid z \in B\}$ is also an invariant Baker domain of $f_{\lambda}$. Clearly, one of $B$ and $\bar{B}$ contains points with positive imaginary parts. Let it be $B$, i.e., $\Im(z)>0$ for each $z \in B$.

We assert that the set $\{\Im(z) \mid z \in B\}$ is unbounded. To see it, suppose on the contrary that $\{\Im(z) \mid z \in B\}$ is bounded. Then $\{\Re(z) \mid z \in B\}$ must be unbounded as $B$ is itself unbounded. Now, let $\left\{z_{k}\right\}_{k>0}$ be a sequence in $B$ such that $\lim _{k \rightarrow \infty}\left|\Re\left(z_{k}\right)\right|=\infty$. Then

$$
f_{\lambda}\left(z_{k}\right)=\frac{\lambda 2^{m} z_{k}^{m}}{\left(e^{z_{k}}-e^{-z_{k}}\right)^{m}} \rightarrow 0 \quad \text { as } k \rightarrow \infty
$$

by Remark 2.1. The point 0 is in the attracting or parabolic domain for each $\lambda>0$ by Remark 3.2. Let $N(0)$ be a neighbourhood of $z=0$ completely lying in the Fatou set. Then, there is a natural number $\hat{k}$ such that $f_{\lambda}\left(z_{k}\right) \in N(0)$ for all $k>\hat{k}$. Consequently, $z_{k}$ is in a Fatou component $U$ such that $f_{\lambda}(U)$ is contained in an attracting domain or a parabolic domain and hence, not in $B$ for $k>\hat{k}$. It contradicts the invariance of $B$. Thus the set $\{\Im(z) \mid z \in B\}$ is unbounded.

Let $B$ be in the first quadrant of the plane. If $B$ is in the second quadrant, the proof follows similarly. For $\theta \in(0,(\pi / 2))$, let $S_{\theta}=\{z \in \mathbb{C} \mid \theta<\operatorname{Arg}(z)<\pi / 2\}$ and $S_{\theta^{\prime}}=\{z \in \mathbb{C} \mid 0<\operatorname{Arg}(z) \leq \theta\}$ where $0<\operatorname{Arg}(z)<2 \pi$. Let $L_{k}=\{z \in \mathbb{C} \mid \Im(z)=\pi k\}$ and $L_{k}^{+}=\left\{z \in L_{k} \mid \Re(z)>0\right\}$ for $k \in \mathbb{Z}$. We now show that the set $\left\{\Im(z) \mid z \in B \cap S_{\theta}\right\}$ is unbounded for each $\theta \in(0, \pi / 2)$. In view of the conclusion obtained in the previous paragraph, it is sufficient to prove that the set $\left\{\Im(z) \mid z \in B \cap S_{\theta^{\prime}}\right\}$ is bounded. Suppose the set $\left\{\Im(z) \mid z \in B \cap S_{\theta^{\prime}}\right\}$ is unbounded for some $\theta$. Then a sequence $\left\{s_{n}\right\}_{n>0}$ of points can be found in $B \cap S_{\theta^{\prime}}$ such that $\Im\left(s_{n}\right) \leq(\tan \theta) \Re\left(s_{n}\right)$ for all $n \in \mathbb{N}$ and $\Im\left(s_{n}\right) \rightarrow \infty$ as $n \rightarrow \infty$. Consequently, $\Re\left(s_{n}\right) \rightarrow \infty$ and

$$
\left|\frac{s_{n}}{\sinh \left(s_{n}\right)}\right| \leq 2 \frac{\left|\Re\left(s_{n}\right)+i \Im\left(s_{n}\right)\right|}{e^{\Re\left(s_{n}\right)}-e^{-\Re\left(s_{n}\right)}} \leq 2 \frac{\left|(1+\tan \theta) \Re\left(s_{n}\right)\right|}{e^{\Re\left(s_{n}\right)}-e^{-\Re\left(s_{n}\right)}} \rightarrow 0 \quad \text { as } n \rightarrow \infty .
$$

It follows that there is an $n_{0} \in \mathbb{N}$ such that $f_{\lambda}\left(s_{n}\right) \in N(0)$ for $n>n_{0}$. Consequently, the set $\left\{s_{n} \mid n>n_{0}\right\}$ is not in the Baker domain, which is a contradiction. Therefore, the set $\left\{\Im(z) \mid z \in B \cap S_{\theta^{\prime}}\right\}$ is bounded, and hence the set $\left\{\Im(z) \mid z \in B \cap S_{\theta}\right\}$ is unbounded. Furthermore, $B \cap S_{\theta}$ has an unbounded connected subset. In particular, there exists an integer $k_{0}$ such that the set $B \cap S_{\theta}$ intersects $L_{k}^{+}$for all $k \geq k_{0}$. Choose $\theta$ in such a way that for all $\delta, \beta \in(\theta, \pi / 2),|m(\delta-\beta)|<(\pi / 4)$ where $f_{\lambda}(z)=\lambda\left(z^{m} / \sinh ^{m} z\right)$.

Case 1. $m$ is odd.

Note that

$$
f_{\lambda}(x+i \pi k)=\lambda \frac{(x+i \pi k)^{m}}{\sinh ^{m}(x+i \pi k)}= \begin{cases}-\lambda \frac{(x+i \pi k)^{m}}{\sinh ^{m} x} & \text { for odd } k, \\ \lambda \frac{(x+i \pi k)^{m}}{\sinh ^{m} x} & \text { for even } k .\end{cases}
$$

Let $z_{1}=x_{1}+i \pi k, z_{2}=x_{2}+i \pi(k+1) \in B \cap S_{\theta}$ for some $k \geq k_{0}$. If $\operatorname{Arg}\left(z_{1}\right)=\theta_{1}$ and $\operatorname{Arg}\left(z_{2}\right)=\theta_{2}$ then $\theta_{1}, \theta_{2} \in(\theta, \pi / 2)$ and $\left|\operatorname{Arg}\left(z_{1}^{m}\right)-\operatorname{Arg}\left(z_{2}^{m}\right)\right|=\left|m\left(\theta_{1}-\theta_{2}\right)\right|<\pi / 4$. Therefore, the two points $z_{1}^{m}$ and $z_{2}^{m}$ belong either to the same quadrant or to two consecutive quadrants. This means either the real parts or the imaginary parts of $z_{1}^{m}$ 
and $z_{2}^{m}$ have same sign. Let the first possibility hold i.e., $\left(\Re\left(z_{1}^{m}\right) / \Re\left(z_{2}^{m}\right)\right)>0$. One of $k$ and $k+1$ is even and the other is odd. Also note that $\left(\lambda / \sinh ^{m} x\right)>0$ for $x>0$. Using equation (4), we have $\Re\left(f_{\lambda}\left(z_{1}\right)\right) / \Re\left(f_{\lambda}\left(z_{2}\right)\right)<0$. In other words, $\Re\left(f_{\lambda}\left(z_{1}\right)\right)$ and $\Re\left(f_{\lambda}\left(z_{2}\right)\right)$ have opposite sign. Thus $f_{\lambda}(B)=B$ intersects the imaginary axis which contradicts Remark 3.3. For $\Im\left(z_{1}^{m}\right) / \Im\left(z_{2}^{m}\right)>0$, arguing similarly, we can get $\Im\left(f_{\lambda}\left(z_{1}\right)\right) / \Im\left(f_{\lambda}\left(z_{2}\right)\right)<$ 0 , which also results in a similar contradiction to Remark 3.3.

Case 2. $m / 2$ is odd.

Note that

$$
\sinh ^{m}\left(x+i\left(\frac{\pi}{2}+2 \pi k\right)\right)=-\cosh ^{m} x \quad \text { for } k \in \mathbb{N} .
$$

Since the line

$$
L_{(\pi / 2)+2 \pi k}=\left\{z \in \mathbb{C} \mid \Im(z)=\frac{\pi}{2}+2 \pi k\right\}
$$

intersects $B \cap S_{\theta}$ for all sufficiently large $k$, there is an even $k^{\prime} \in \mathbb{N}$ such that the points $z_{3}=x_{3}+i\left((\pi / 2)+2 \pi k^{\prime}\right)$ and $z_{4}=x_{4}+i\left(2 \pi k^{\prime}\right)$ are in $B \cap S_{\theta}$ for some $x_{3}, x_{4}>0$ where $\theta$ is so chosen that $\left|\operatorname{Arg}\left(z_{3}^{m}\right)-\operatorname{Arg}\left(z_{4}^{m}\right)\right|<\pi / 4$. Now,

$$
f_{\lambda}\left(z_{3}\right)=-\lambda \frac{\left(x_{3}+i\left((\pi / 2)+2 \pi k^{\prime}\right)\right)^{m}}{\cosh ^{m} x_{3}} \quad \text { and } \quad f_{\lambda}\left(z_{4}\right)=\lambda \frac{\left(x_{4}+i 2 \pi k^{\prime}\right)^{m}}{\sinh ^{m} x_{4}} .
$$

Arguing exactly in the same manner as in Case 1 , it is found that either

$$
\frac{\Re\left(f_{\lambda}\left(z_{3}\right)\right)}{\Re\left(f_{\lambda}\left(z_{4}\right)\right)}<0 \quad \text { or } \quad \frac{\Im\left(f_{\lambda}\left(z_{3}\right)\right)}{\Im\left(f_{\lambda}\left(z_{4}\right)\right)}<0 .
$$

Both of these possibilities contradict Remark 3.3.

Therefore, the Fatou set of $f_{\lambda}$ does not contain any Baker domain.

THEOREM 4.2. Let $f_{\lambda} \in \mathcal{S}$ and $\lambda>0$. Then, the Fatou set of $f_{\lambda}$ has no wandering domain.

Proof. By Remark 3.2, the set $P\left(f_{\lambda}\right) \backslash\{\infty\}$ is in the Fatou set of $f_{\lambda}$. Since $\infty$ is in the derived set $P\left(f_{\lambda}\right)^{\prime}$ of $P\left(f_{\lambda}\right)$, we have $\mathcal{J}\left(f_{\lambda}\right) \cap P\left(f_{\lambda}\right)^{\prime}=\{\infty\}$. If a point $z_{0}$ is in a wandering domain of $f_{\lambda}$ then, every limit point of $\left\{f_{\lambda}^{n}\left(z_{0}\right)\right\}_{n>0}$ is infinity [22]. Since $S_{2}\left(f_{\lambda}\right)$ is bounded, $f_{\lambda}^{2 n}\left(z_{0}\right)$ does not tend to infinity as $n \rightarrow \infty$. Then, we can find a subsequence $\left\{n_{k}\right\}_{k>0}$ of $\{2 n\}_{n>0}$ such that $\left\{f_{\lambda}^{n_{k}}\left(z_{0}\right)\right\}_{k>0}$ is bounded. Let us consider $\left\{f_{\lambda}^{n_{k}}\right\}_{k>0}$. Since $\left\{f_{\lambda}^{n}\right\}_{n>0}$ is normal at $z_{0}$, there is a subsequence $\left\{f_{\lambda}^{n_{k, m}}\right\}_{m>0}$ of $\left\{f_{\lambda}^{n_{k}}\right\}_{k>0}$ such that $\lim _{m \rightarrow \infty} f_{\lambda}^{n_{k, m}}\left(z_{0}\right)=\infty$. However, it is not possible because $\left\{n_{k, m}\right\}_{m>0}$ is a subsequence of $\left\{n_{k}\right\}_{k>0}$. Therefore, the Fatou set of $f_{\lambda}$ does not contain any wandering domain.

THEOREM 4.3. Let $f_{\lambda} \in \mathcal{S}$ and $\lambda>0$.

(1) For $\lambda<\lambda^{*}$, the Fatou set $\mathcal{F}\left(f_{\lambda}\right)$ of $f_{\lambda}$ is the basin of attraction of the unique real attracting fixed point $a_{\lambda}$ of $f_{\lambda}$.

(2) For $\lambda=\lambda^{*}$, the Fatou set $\mathcal{F}\left(f_{\lambda}\right)$ of $f_{\lambda}$ is the parabolic basin corresponding to the unique real rationally indifferent fixed point $x^{*}$ of $f_{\lambda}$.

(3) For $\lambda>\lambda^{*}$, the Fatou set $\mathcal{F}\left(f_{\lambda}\right)$ of $f_{\lambda}$ is the basin of attraction or parabolic basin corresponding to a cycle of real 2-periodic points $\left\{a_{1 \lambda}, a_{2 \lambda}\right\}$ of $f_{\lambda}$ 
Proof. We know that the boundary of any rotational domain of a meromorphic function $f$ is contained in the closure of the set $P(f)$ [4]. Thus, the Fatou set of $f_{\lambda}$ does not contain any rotational domain. By Theorems 4.1 and 4.2, the Fatou set of $f_{\lambda}$ also does not contain any Baker domain and wandering domain for $\lambda>0$.

If $U$ is an attracting domain or parabolic domain of period $p$ and $z_{u}$ is the corresponding attracting or rationally indifferent periodic point of $f_{\lambda}$, then there is a singular value $s$ of $f_{\lambda}$ such that $f_{\lambda}^{n p}\left(f_{\lambda}^{k}(s)\right) \rightarrow z_{u}$ as $n \rightarrow \infty$ for some $k, 0<k \leq p$. Since all the singular values and their forward orbits (whenever defined) are in $\mathbb{R}, z_{u}$ is real. Therefore, any attracting or parabolic domain of $f_{\lambda}$ corresponds to a real attracting or rationally indifferent periodic point.

(1) For $0<\lambda<\lambda^{*}, f_{\lambda}$ has only one real periodic point which is the attracting fixed point $a_{\lambda}$. Therefore, $\mathcal{F}\left(f_{\lambda}\right)$ is the basin of attraction of $a_{\lambda}$.

(2) For $\lambda=\lambda^{*}, f_{\lambda}$ has only one real periodic point which is the rationally indifferent fixed point $x^{*}$. Therefore, $\mathcal{F}\left(f_{\lambda}\right)$ is the parabolic basin corresponding to $x^{*}$.

(3) For $\lambda>\lambda^{*}, f_{\lambda}$ has a repelling fixed point $r_{\lambda}$ and a cycle of real 2-periodic points $\left\{a_{1 \lambda}, a_{2 \lambda}\right\}$ which is either attracting or rationally indifferent. Therefore, $\mathcal{F}\left(f_{\lambda}\right)$ is the attracting basin or parabolic basin corresponding to $\left\{a_{1 \lambda}, a_{2 \lambda}\right\}$.

Since $f_{\lambda}$ and $f_{-\lambda}$ are conformally conjugate, the dynamics of $f_{\lambda}$ for $\lambda<0$ is as follows.

COROLlaRY 4.1. Let $f_{\lambda} \in \mathcal{S}$ and $\lambda<0$.

(1) For $-\lambda^{*}<\lambda<0$, the Fatou set $\mathcal{F}\left(f_{\lambda}\right)$ of $f_{\lambda}$ is the basin of attraction of the unique real attracting fixed point of $f_{\lambda}$.

(2) For $\lambda=-\lambda^{*}$, the Fatou set $\mathcal{F}\left(f_{\lambda}\right)$ of $f_{\lambda}$ is the parabolic basin corresponding to the unique real rationally indifferent fixed point of $f_{\lambda}$.

(3) For $\lambda<-\lambda^{*}$, the Fatou set $\mathcal{F}\left(f_{\lambda}\right)$ of $f_{\lambda}$ is the basin of attraction or parabolic basin corresponding to a cycle of real 2-periodic points of $f_{\lambda}$.

\section{Topology of Fatou components}

Topology of the Fatou components of $f_{\lambda}, \lambda>0$ is investigated in this section. It is observed from Theorem 4.3 that the Fatou set of $f_{\lambda}$ contains components with period one and two. The connectivity of a periodic Fatou component of a meromorphic function is either one, two or infinity whereas the connectivity of a pre-periodic Fatou component can be any finite number [2]. In Theorem 5.1, it is proved that the Fatou set of $f_{\lambda}, 0<\lambda<\lambda^{*}$ is infinitely connected. The existence of pre-periodic Fatou components is established and the connectivity of all the Fatou components of $f_{\lambda}$ is determined for $\lambda>\lambda^{*}$ in Theorem 5.2.

THEOREM 5.1. Let $f_{\lambda} \in \mathcal{S}$ and $0<\lambda<\lambda^{*}$. Then, the Fatou set $\mathcal{F}\left(f_{\lambda}\right)$ of $f_{\lambda}$ is connected. Furthermore, the Fatou set $\mathcal{F}\left(f_{\lambda}\right)$ is infinitely connected.

Proof. By Theorem 3.2(1), $\lim _{n \rightarrow \infty} f_{\lambda}^{n}(x)=a_{\lambda}$ for $x \in \mathbb{R}$ and $0<\lambda<\lambda^{*}$ where $a_{\lambda}$ is the attracting fixed point of $f_{\lambda}$. The Fatou set of $f_{\lambda}$ is the attracting basin

$$
A\left(a_{\lambda}\right)=\left\{z \in \mathbb{C} \mid f_{\lambda}^{n}(z) \rightarrow a_{\lambda} \text { as } n \rightarrow \infty\right\} \text { for } 0<\lambda<\lambda^{*} .
$$


Let $I\left(a_{\lambda}\right)$ be the immediate basin of attraction of $a_{\lambda}$. By definition, $I\left(a_{\lambda}\right)$ is a forward invariant connected subset of the Fatou set $\mathcal{F}\left(f_{\lambda}\right)$. Note that $A\left(a_{\lambda}\right)=I\left(a_{\lambda}\right)$ if $I\left(a_{\lambda}\right)$ is backward invariant. Since $I\left(a_{\lambda}\right)$ is connected, in order to prove the connectedness of $\mathcal{F}\left(f_{\lambda}\right)$, it is sufficient to show that $I\left(a_{\lambda}\right)$ is backward invariant.

Let, if possible, $V$ be a component of $f_{\lambda}^{-1}\left(I\left(a_{\lambda}\right)\right)$ different from $I\left(a_{\lambda}\right)$. Since 0 is an omitted value of $f_{\lambda}$, each singularity of $f_{\lambda}^{-1}$ lying over 0 is transcendental. It means that $V$ contains an asymptotic path $\gamma$ corresponding to the asymptotic value 0 and by Remark 2.1, the set $\{\Re(z) \mid z \in \gamma\}$ is unbounded. Therefore, the set $\{\Re(z) \mid z \in V\}$ is unbounded. The function $f_{\lambda}$ is even and $f_{\lambda}(\bar{z})=\overline{f_{\lambda}(z)}$ for all $z \in \mathbb{C}$. In view of Remark 3.3, it is assumed without loss of generality that, the set $V$ is in the upper half plane $\{z \in \mathbb{C} \mid \Im(z)>0\}$. Let $\left\{w_{n}\right\}_{n>0}$ be a sequence on $\gamma$ such that $\Re\left(w_{n}\right) \rightarrow \infty$ as $n \rightarrow \infty$. Then $\lim _{n \rightarrow \infty} f_{\lambda}\left(w_{n}\right)=0$. Each of the vertical lines $l_{n}=\left\{z \in \mathbb{C} \mid \mathfrak{N}(z)=\Re\left(w_{n}\right)\right.$ and $\left.0 \leq \Im(z)<\Im\left(w_{n}\right)\right\}$ joins a point of $V$ and a point of $\mathbb{R} \cap I\left(a_{\lambda}\right)$ and we get that $l_{n}$ intersects the boundary $\partial V$ of $V$ for each $n$. Let $z_{n} \in l_{n} \cap \partial V$. Then $z_{n} \in \mathcal{J}\left(f_{\lambda}\right)$ and $\Im\left(z_{n}\right)<\Im\left(w_{n}\right)$ for all $n$. Furthermore,

$$
\begin{aligned}
\left|f_{\lambda}\left(z_{n}\right)\right| & =\lambda\left\{\left(\frac{\Re\left(z_{n}\right)^{2}+\Im\left(z_{n}\right)^{2}}{\sinh ^{2} \Re\left(z_{n}\right)+\sin ^{2} \Im\left(z_{n}\right)}\right)^{1 / 2}\right\}^{m} \\
& <\lambda\left\{\left(\frac{\Re\left(w_{n}\right)^{2}+\Im\left(w_{n}\right)^{2}}{\sinh ^{2} \Re\left(w_{n}\right)+\sin ^{2} \Im\left(z_{n}\right)}\right)^{1 / 2}\right\}^{m} .
\end{aligned}
$$

Since the sequence $\left\{\sin ^{2}\left(\Im\left(z_{n}\right)\right)\right\}_{n>0}$ is bounded, the right-hand side of equation (5) is equal to $\left|f_{\lambda}\left(w_{n}\right)\right|$ when $n \rightarrow \infty$. Therefore, $\lim _{n \rightarrow \infty} f_{\lambda}\left(z_{n}\right)=0$. Let $D_{r}(0)=\{z \in \mathbb{C}$ : $|z|<r\} \subset I\left(a_{\lambda}\right)$. Then, there exists an $n_{0}$ such that $f_{\lambda}\left(z_{n}\right) \in D_{r}(0)$ for all $n>n_{0}$. It means that $z_{n}$ is in the Fatou set of $f_{\lambda}$ for $n>n_{0}$, which is a contradiction. Therefore, each component of $f_{\lambda}^{-1}\left(I\left(a_{\lambda}\right)\right)$ intersects $I\left(a_{\lambda}\right)$ and hence is a subset of $I\left(a_{\lambda}\right)$. Thus $I\left(a_{\lambda}\right)$ is backward invariant.

Since $\mathcal{F}\left(f_{\lambda}\right)$ is connected and contains an attracting fixed point, it is invariant. The connectivity of any invariant Fatou component of a meromorphic function is one, two or infinity, two being the case when the component is an Herman ring. Since the Fatou set $\mathcal{F}\left(f_{\lambda}\right)$ is an attracting domain for $0<\lambda<\lambda^{*}$, the connectivity of $\mathcal{F}\left(f_{\lambda}\right)$ is either one or infinity. If possible, let $\mathcal{F}\left(f_{\lambda}\right)$ be simply connected. Then, the Julia set $\mathcal{J}\left(f_{\lambda}\right)$ is connected. As the point at infinity and a pole $w^{*}$ lying on the imaginary axis are in $\mathcal{J}\left(f_{\lambda}\right)$, there is an unbounded connected subset $J_{w^{*}}$ of the Julia set containing $w^{*}$. Now, $\overline{-J_{w^{*}}}=\{z \in \mathbb{C} \mid$ $\left.-\bar{z} \in J_{w^{*}}\right\}$ is also in the Julia set by Proposition 2.1. Thus $J=J_{w^{*}} \cup \overline{-J_{w^{*}}}$ is in the Julia set and the set $\widehat{\mathbb{C}} \backslash J$ has at least two components each intersecting the Fatou set of $f_{\lambda}$. This contradicts the fact that $\mathcal{F}\left(f_{\lambda}\right)$ is connected. Therefore, $\mathcal{F}\left(f_{\lambda}\right)$ is infinitely connected for $0<\lambda<\lambda^{*}$.

Remark 5.1. Since the Fatou set is connected with connectivity greater than three for $0<\lambda<\lambda^{*}$, singleton components of $\mathcal{J}\left(f_{\lambda}\right)$ are dense in $\mathcal{J}\left(f_{\lambda}\right)[\mathbf{1 0}]$.

It is seen in Theorem 5.1 that the Fatou set of $f_{\lambda}$ is connected and hence unbounded for $0<\lambda<\lambda^{*}$. The next proposition shows that there are at least three Fatou components of $f_{\lambda}$, two of which are unbounded for $\lambda>\lambda^{*}$. 
Proposition 5.1. Let $f_{\lambda} \in \mathcal{S}$ and $\lambda>\lambda^{*}$. If $U^{+}, U^{-}$and $U_{0}$ denote the Fatou components containing $\left(a_{2 \lambda},+\infty\right),\left(-\infty,-a_{2 \lambda}\right)$ and 0 respectively where $\left\{a_{1 \lambda}, a_{2 \lambda}\right\}$ is the 2-cycle of real periodic points of $f_{\lambda}$, then the Fatou components $U^{+}, U^{-}$and $U_{0}$ are mutually disjoint. Further, the components $U^{+}$and $U^{-}$are unbounded.

Proof. Observe that both $U^{+}$and $U^{-}$are mapped into $U_{0}$ and $U_{0}$ is mapped into $U^{+}$ by $f_{\lambda}$ for $\lambda>\lambda^{*}$. Since $U_{0}$ and $U^{+}$form a cycle of 2-periodic Fatou components, $U_{0} \neq U^{+}$. If $U_{0}$ intersects $U^{-}$then $U_{0}=U^{-}$will become invariant, which is not true. Therefore, $U_{0}$ is different from $U^{+}$and $U^{-}$. If $U^{+}$and $U^{-}$are the same component of $\mathcal{F}\left(f_{\lambda}\right)$ then $U^{+}=U^{-}$intersects the imaginary axis. Then, since all the points in the imaginary axis are mapped onto $\mathbb{R} \backslash(-\lambda, \lambda) \subset\left(-\infty,-a_{2 \lambda}\right) \cup\left(a_{2 \lambda},+\infty\right)$, the points of the set $U^{+} \cap\{i y \mid y \in \mathbb{R}\}$ are mapped into $U^{+}$and consequently, $U^{+}$is invariant, leading to a contradiction. Therefore, $U_{0}, U^{+}$and $U^{-}$are mutually disjoint components of $\mathcal{F}\left(f_{\lambda}\right)$ for $\lambda>\lambda^{*}$. The components $U^{-}$and $U^{+}$are unbounded by definition.

THEOREM 5.2. Let $f_{\lambda} \in \mathcal{S}$ and $\lambda>\lambda^{*}$. Then, the Fatou set $\mathcal{F}\left(f_{\lambda}\right)$ of $f_{\lambda}$ contains infinitely many pre-periodic components and each component of $\mathcal{F}\left(f_{\lambda}\right)$ is simply connected.

Proof. It is clear from Theorem 3.2 that the point $0 \in \mathcal{F}\left(f_{\lambda}\right)$ for all $\lambda$. Let $U_{0}$ be the Fatou component containing 0 . If $\lambda>\lambda^{*}$ and $\left\{a_{1 \lambda}, a_{2 \lambda}\right\}$ is the 2-cycle of real periodic points of $f_{\lambda}$ then by Theorem 3.2, $\left(-\infty,-a_{2 \lambda}\right)$ and $\left(a_{2 \lambda},+\infty\right)$ are in the Fatou set of $f_{\lambda}$. Let $U^{-}$and $U^{+}$be the Fatou components of $f_{\lambda}$ containing $\left(-\infty,-a_{2 \lambda}\right)$ and $\left(a_{2 \lambda},+\infty\right)$ respectively. If a pre-image of a point of $U^{-}$lies in $U^{-}$then $U^{-} \cap f_{\lambda}\left(U^{-}\right) \neq \emptyset$ which shows that $U^{-}=f_{\lambda}\left(U^{-}\right)$since $f_{\lambda}\left(U^{-}\right)$is connected. It means that $U^{-}$is forward invariant. But it is already known that $U^{-}$is not forward invariant. Therefore, no preimage of any point of $U^{-}$lies in $U^{-}$. In other words, $U^{-}$is not backward invariant. Since none of $U_{0}$ and $U^{+}$is mapped into $U^{-}$by $f_{\lambda}$, each component of $f_{\lambda}^{-1}\left(U^{-}\right)$is different from $U_{0}$ and $U^{+}$, and consequently is a pre-periodic Fatou component. Repeating the same arguments for each component of $f_{\lambda}^{-1}\left(U^{-}\right)$and continuing the process, we can find infinitely many pre-periodic Fatou components.

Let $U$ be any Fatou component of $f_{\lambda}$. Suppose, on the contrary that $U$ is multiply connected. Let $\gamma$ be a simple closed curve in $U$ such that the bounded component $B\left(\gamma^{c}\right)$ of $\gamma^{c}=\widehat{\mathbb{C}} \backslash \gamma$ intersects the Julia set $\mathcal{J}\left(f_{\lambda}\right)$. Set $B_{j}=f_{\lambda}^{j}\left(B\left(\gamma^{c}\right)\right)$ for $j \in \mathbb{N}$. If $B\left(\gamma^{c}\right)$ does not contain a pole of $f_{\lambda}$ then $f_{\lambda}(z)$ is analytic on $\overline{B\left(\gamma^{c}\right)}$, the closure of $B\left(\gamma^{c}\right)$, and $B_{1}=f_{\lambda}\left(B\left(\gamma^{c}\right)\right)$ is bounded. Further, the function $f_{\lambda}(z)$ maps the interior of $B\left(\gamma^{c}\right)$ (which intersects the Julia set) into the interior of $B_{1}$ and, by the complete invariance of $\mathcal{J}\left(f_{\lambda}\right)$, it follows that $B_{1} \cap \mathcal{J}\left(f_{\lambda}\right) \neq \emptyset$. If $B_{1}$ does not contain any pole of $f_{\lambda}$ then consider $B_{2}=f_{\lambda}\left(B_{1}\right)$ and repeat the above arguments. Since the pre-images of all the poles of $f_{\lambda}$ are dense in $\mathcal{J}\left(f_{\lambda}\right), B\left(\gamma^{c}\right)$ contains a point $\tilde{w}$ such that $f_{\lambda}^{n}(\tilde{w})$ is a pole of $f_{\lambda}$ for a natural number $n$. Let $n^{*}$ the minimum of all such natural numbers, minimum being taken over all points in the backward orbit of $\infty$ which lie in $B\left(\gamma^{c}\right)$. Then, the set $B_{n^{*}}$ contains a pole. Since all the poles of $f_{\lambda}$ are on the imaginary axis, the boundary of $B_{n^{*}}$ intersects the imaginary axis. Therefore, the set $B_{n^{*}+1}=f_{\lambda}\left(B_{n^{*}}\right)$ contains a neighbourhood of $\infty$ and the unboundedness of $U^{+}$and $U^{-}$gives that $B_{n^{*}+1}$ intersects both $U^{+}$and $U^{-}$. Since $f_{\lambda}(i y) \in \mathbb{R}$ and $\left|f_{\lambda}(i y)\right| \geq \lambda$ for all $y \in \mathbb{R}$, the $f_{\lambda}$-image of $\partial B_{n}^{*}$ intersects at least one of $U^{+}$or $U^{-}$. Note that $\partial B_{j+1} \subseteq f_{\lambda}\left(\partial B_{j}\right)$ for $j=1,2,3, \ldots, n^{*}$. 
TABLE 1. Comparison between the dynamics of $\lambda \tanh \left(e^{z}\right)$ and $\lambda\left(z^{m} / \sinh ^{m} z\right)$.

\begin{tabular}{ll}
\hline & $\begin{array}{l}\text { Dynamics of } f_{\lambda}(z)=\lambda z^{m} / \sinh ^{m} z, \lambda \neq 0, \\
\text { where } m \text { or } m / 2 \text { is an odd natural number }\end{array}$ \\
\hline$g_{\lambda}$ is periodic with period $2 \pi i$. & $f_{\lambda}$ is not periodic. \\
$g_{\lambda}$ is neither even nor odd. & $f_{\lambda}$ is even. \\
$g_{\lambda}$ has no critical values. & $f_{\lambda}$ has infinitely many critical values. \\
$g_{\lambda}$ has three (finite) asymptotic values $0, \lambda$ & $f_{\lambda}$ has only one (finite) asymptotic value 0. \\
and $-\lambda$. & \\
The set of all singular values of $g_{\lambda}$ is finite. & The set of all singular values of $f_{\lambda}$ is \\
& unbounded. \\
Bifurcation in the dynamics of $g_{\lambda}$ occurs at & Bifurcation in the dynamics of $f_{\lambda}$ occurs \\
one critical parameter $\lambda^{*} \approx-3.2946$. & at two critical parameters $\pm \lambda^{*}(m)$ whose \\
& values depend on $f$. \\
The Fatou set of $g_{\lambda}$ has infinitely many & The Fatou set of $f_{\lambda}$ has infinitely many \\
components and each component is simply & components and each component is simply \\
connected for $\lambda \leq \lambda^{*}$. & connected for $|\lambda| \geq \lambda^{*}(m)$. \\
The Fatou set of $g_{\lambda}$ is infinitely connected & $\begin{array}{l}\text { The Fatou set of } f_{\lambda} \text { is infinitely connected } \\
\text { for } \lambda>\lambda^{*} .\end{array}$ \\
\hline
\end{tabular}

Therefore, $\partial B_{n^{*}+1} \subseteq f_{\lambda}\left(\partial B_{n^{*}}\right) \subseteq \cdots \subseteq f_{\lambda}^{n^{*}+1}(\gamma) \subset \mathcal{F}\left(f_{\lambda}\right)$ and consequently, $\partial B_{n^{*}+1}$ lies either in $U^{+}$or in $U^{-}$. Since neither $U^{+}$nor $U^{-}$intersects the imaginary axis, $\partial B_{n^{*}+1}$ cannot wind around $U_{0}$. Now, $U_{0}$ is a subset of $B_{n^{*}+1}$ and each singularity of $f_{\lambda}^{-1}$ lying over 0 is transcendental. This means that $B_{n^{*}}$ contains an asymptotic path corresponding to the asymptotic value 0 which contradicts the boundedness of $B_{n^{*}}$. Therefore, $U$ is simply connected.

Remark 5.2. Theorem 5.2 is true for $\lambda=\lambda^{*}$ and the proof is similar.

The function $\lambda\left(z^{m} / \sinh ^{m} z\right)$ differs in many fundamental properties from the meromorphic function $\lambda \tanh \left(e^{z}\right)$, but these functions exhibit similar bifurcations in their dynamics. The iteration of $\lambda \tanh \left(e^{z}\right)$ is studied in [11]. Table 1 provides a comparison between the dynamical properties of these two functions.

Acknowledgement. This work was carried out at IIT Guwahati and was supported by CSIR Senior Research Fellowship Grant No. 9/731(31)2004-EMR-I for the first author.

\section{REFERENCES}

[1] I. N. Baker, J. Kotus and L. Yinian. Iterates of meromorphic functions II: examples of wandering domains. J. London Math. Soc. (2) 2(42) (1990), 267-278.

[2] I. N. Baker, J. Kotus and L. Yinian. Iterates of meromorphic functions III: preperiodic domains. Ergod. Th. \& Dynam. Sys. 11 (1991), 603-618. 
[3] I. N. Baker, J. Kotus and L. Yinian. Iterates of meromorphic functions IV: critically finite functions. Results Math. 22 (1992), 651-656.

[4] W. Bergweiler. Iteration of meromorphic functions. Bull. Amer. Math. Soc. (N.S.) 29(2) (1993), 151-188.

[5] W. Bergweiler. Newton's method and a class of meromorphic functions without wandering domains. Ergod. Th. \& Dynam. Sys. 13 (1993), 231-247.

[6] W. Bergweiler. Singularities in Baker domains. Comput. Methods Funct. Theory 1(1) (2001), 41-49.

[7] W. Bergweiler and A. E. Eremenko. On the singularities of the inverse to a meromorphic function of finite order. Rev. Mat. Iberoam. 11 (1995), 355-373.

[8] W. Bergweiler, M. Haruta, H. Kriete, H. Meier and N. Terglane. On the limit functions of iterates in wandering domains. Ann. Acad. Sci. Fenn. Math. Ser. A. I. Math. 18 (1993), 369-375.

[9] R. L. Devaney and L. Keen. Dynamics of tangent. Dynamical Systems (Lecture Notes in Mathematics, 1342). Springer, Berlin, 1988, pp. 105-111.

[10] P. Domínguez. Dynamics of transcendental meromorphic functions. Ann. Acad. Sci. Fenn. Math. 23 (1998), 225-250.

[11] M. G. P. Prasad and T. Nayak. Dynamics of $\lambda \tanh \left(e^{z}\right)$. Discrete Contin. Dyn. Syst. 19(1) (2007), 121-138.

[12] M. E. Herring. Mapping properties of Fatou components. Ann. Acad. Sci. Fenn. Math. 23 (1998), 263-274.

[13] W. H. Jiang. Dynamics of $\lambda \tan z$. PhD Thesis, Graduate Center of the City University of New York, CUNY, 1991.

[14] L. Keen and J. Kotus. Dynamics of the family $\lambda \tan z$. Conform. Geom. Dyn. 1 (1997), $28-57$.

[15] H. König. Conformal conjugacies in Baker domains. J. London Math. Soc. (2) 59(2) (1999), 153-170.

[16] S. Morosowa. An example of cyclic Baker domains. Mem. Fac. Sci. Kochi. Univ. Ser. A Math. 20 (1999), 123-126.

[17] R. Nevanlinna. Analytic Functions. Springer, Berlin, 1970.

[18] P. J. Rippon and G. M. Stallard. Singularities of meromorphic functions with Baker domains. Math. Proc. Cambridge Philos. Soc. 141 (2006), 371-382.

[19] P. J. Rippon and G. M. Stallard. Iteration of a class of hyperbolic meromorphic functions. Proc. Amer. Math. Soc. 127(11) (1999), 3251-3258.

[20] G. M. Stallard. A class of meromorphic functions with no wandering domains. Ann. Acad. Sci. Fenn. Ser. Math. 16 (1991), 211-226.

[21] J. H. Zheng. On transcendental meromorphic functions which are geometrically finite. J. Aust. Math. Soc. 72 (2002), 93-107.

[22] J. H. Zheng. Singularities and limit functions in iteration of meromorphic functions. J. London Math. Soc. (2) 67(1) (2003), 195-207.

[23] J. H. Zheng. Singularities and wandering domains in iteration of meromorphic functions. Illinois J. Math. 44(3) (2000), 520-530. 